\title{
Corneal Cloudiness: A Presenting Feature of Mucopolysaccharidosis Type I
}

\author{
Surbhi Khurana ${ }^{1} \cdot$ Parul Chawla Gupta $^{1} \cdot$ Ranjan Kumar Behera ${ }^{1} \cdot$ Himanshi Singh ${ }^{1} \cdot$ Jagat Ram ${ }^{1}$
}

Received: 15 May 2021 / Accepted: 10 June 2021 / Published online: 21 July 2021

(c) Dr. K C Chaudhuri Foundation 2021

To the Editor: A 10-y-old 'short-statured' male presented to us with decreased vision in both the eyes since birth. Ocular examination revealed cloudy corneas in both the eyes (Fig. 1a, b). Family history was unremarkable. Suspecting it to be mucopolysaccharidosis, the child was referred to a pediatrician. Systemic examination revealed global developmental delay, short stature, abdominal distension, umbilical hernia, and hepatosplenomegaly with dysmorphic facial features like frontal bossing, saddle nose, wide mouth, and macroglossia (Fig. 1c, d). As the patient had frank corneal clouding, skeletal dysplasia, umbilical hernia, and intellectual impairment, he was most likely to have mucopolysaccharidosis type I, Hurler syndrome [1]. The child was planned for sequential penetrating keratoplasty. For the disorder, the option of enzyme replacement therapy was discussed with the parents, but could not be materialized because of financial constraints.

Mucopolysaccharidoses (MPS) are a group of rare lysosomal storage disorders characterized by accumulation of glycosaminoglycans in various parts of the body. The common clinical presentations are facial and skeletal abnormalities, corneal cloudiness, optic atrophy, pigmentary retinopathy, myocardial involvement and hepatosplenomegaly [2]. Ocular manifestations are common in MPS I, VI, and VII. MPS $\mathrm{I}$ is an autosomal recessive disorder with three phenotypic subtypes-Hurler, Hurler-Scheie, and Scheie; Hurler being the most severe [3]. MPS is a rare cause for bilateral corneal haziness and should be considered as a differential diagnosis for the same in children. Treatment of these disorders usually requires a multidisciplinary approach.

Jagat Ram

drjagatram@gmail.com

1 Department of Ophthalmology, Advanced Eye Centre, Post Graduate Institute of Medical Education and Research, Chandigarh 160012, India
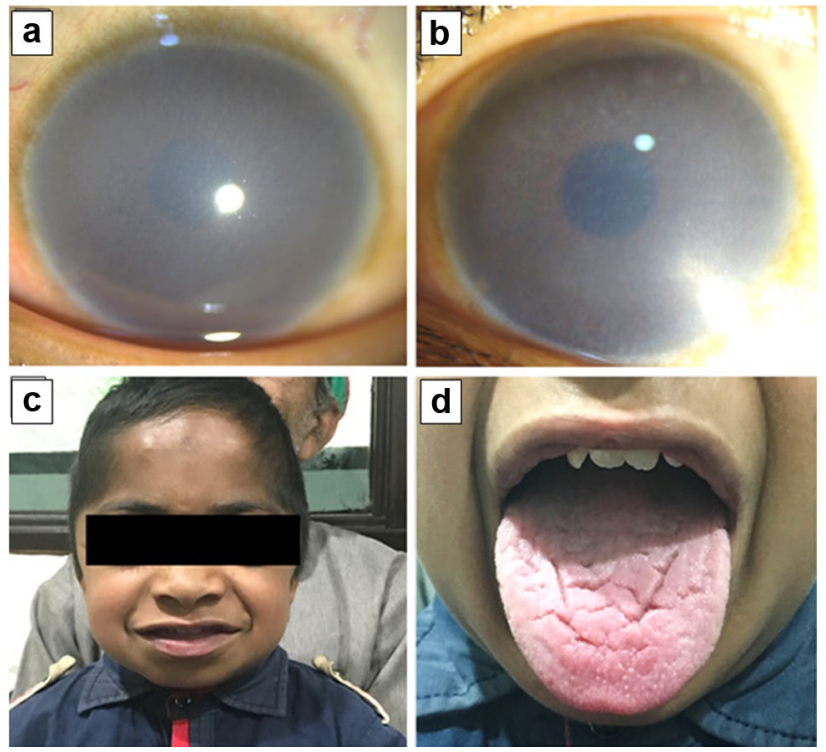

Fig. 1 a Anterior segment photograph of the right eye showing corneal cloudiness $\mathbf{b}$ Anterior segment photograph of the left eye showing corneal cloudiness $\mathbf{c}$ Photograph showing dysmorphic facies, like frontal bossing, saddle nose, wide mouth and macroglossia, suggestive of mucopolysaccharidosis $\mathbf{d}$ Photograph showing macroglossia

\section{Declarations}

Conflict of Interest None.

\section{References}

1. Fenzl CR, Teramoto K, Moshirfar M. Ocular manifestations and management recommendations of lysosomal storage disorders I: mucopolysaccharidoses. Clin Ophthalmol. 2015;9:1633-44.

2. Ashworth JL, Biswas S, Wraith E, et al. Mucopoly-saccharidoses and the eye. Surv Ophthalmol. 2006;51:1-17

3. Beck M, Arn P, Giugliani R, Muenzer J, Okuyama T, Taylor J. The natural history of MPS I: global perspectives from the MPS I registry. Genet Med. 2014;16:759-65.

Publisher's Note Springer Nature remains neutral with regard to jurisdictional claims in published maps and institutional affiliations. 